\title{
An XAFS Study on the Electronic Structure Study of the Boron Substituted $\mathrm{CuFeO}_{2}$
}

\author{
Osman Murat Ozkendir
}

Department of Natural and Mathematical Sciences, Faculty of Engineering, Tarsus University, Tarsus, Turkey E-mail address: ozkendir@tarsus.edu.tr

\author{
ORCID numbers of authors: \\ 0000-0002-0810-9938
}

Received date: 05.01 .2021

Accepted date: 08.03.2021

\begin{abstract}
$\mathrm{CuFeO}_{2}$ is a well known antiferromagnetic material with its geometrically frustrated antiferromagnetic (AFM) $\left[T_{N}=11 \mathrm{~K}\right]$ crystal. Besides, delafossite $\mathrm{CuFeO}_{2}$ oxide crystal is known to be nonstoichiometric under the influence of oxygen as a result of the change in cation valence bands. In this study, boron atoms were substituted in the Fe coordination and the electronic response on irons valence band is probed. Due to the high difference in the ionic radii of the host and substituted atoms, different crystal structure formation was expected. However, calculations showed that boron atoms tend to locate in Fe coordination and preferred to be part of the host crystal by bonding with the oxygen atoms. In addition, the presence of the light boron atoms was determined to weaken the scattering intensities which cause a longer mean free path for the photoelectrons which means better conductivity of the material.
\end{abstract}

Keywords: Absorption; XAFS; Electronic Structure; Crystal Structure

\section{Introduction}

Transition metals have vast application in current technology due to their interesting electronic interactions with other metals that make them an active role player in molecular interplays. Their desired electronic and magnetic properties make them popular for many scientific studies such as their superior conductivity properties, magnetic sequences as well as their semiconductor properties (magnetic semiconductors). Also, the $3 \mathrm{~d}$ elements are abundant in the earth's crust, and that means the commercially available powders are inexpensive and easy to use [1-5]. That's one of the reasons why the most popular magnetic materials of interest in the technological field are the ferromagnetic $3 \mathrm{~d}$ transition metals, i.e., $\mathrm{Fe}, \mathrm{Co}$, and $\mathrm{Ni}$, and their oxides. Low dimensional transition metal oxide $\mathrm{CuFeO}_{2}$ is one of the popular members of the delafossite $\mathrm{ABO}_{2}$ compounds, which is also one of the leading antiferromagnetic iron oxides with rhombohedral geometry and " R-3m" space group $\left[\mathrm{T}_{\mathrm{N}} \pm 11 \mathrm{~K}\right)$ [6-9]. Delafossite $\mathrm{CuFeO}_{2}$ is a remarkable magnetic material with its broken magnetic structure. The atomic structure of the delafossite group consists of a sheet of linearly coordinated "A" cations stacked between edge-shared octahedral layers $\left(\mathrm{BO}_{6}\right)$. 
Studies on materials require a good scientific background and the right selection of the most appropriate techniques for the study. There are different techniques chosen to probe the different properties of the materials. But, one of the technique became popular with its useful data that can be used for several purposes, mainly for electronic structure and crystal structure, is the X-ray Absorption Fine Structure (XAFS) Spectroscopy. XAFS is a synchrotron radiation based technique and it can yield rich data that support the analysis of both crystal and electronic structure properties. The technique is one of the best tool to collect data on both single crystalline, polycrystalline, and non-crystalline materials. The XAFS data can be analyzed in two parts. The first part is called as the XANES (X-ray Absorption NearEdge Spectroscopy) and the EXAFS (Extended-XAFS). Data of the XANES region on the XAFS spectra provide information about the electronic structure of the absorbing atom in the material and the bonding properties with the neighboring atoms. The XANES region lies roughly $20-30 \mathrm{eV}$ below and 40-50 eVabove the main absorption edge. While, $30-40 \mathrm{eV}$ beyond the XANES region is calledNear Edge XAFS (NEXAFS), which has the strong multiple scattering tracks of the excited photoelectrons, the region beyond NEXAFS is called the Extended-XAFS (EXAFS).

The EXAFS region is due to the interference of the excited photoelectrons' incoming and outgoing wavefunctions after the scattering process from a nearest neighboring atom. The scattering mechanism is addressed by the EXAFS "chi” $(\chi)$ equation;

$$
\chi=\left[\mu(E)-\mu_{o}(E)\right] / \Delta \mu_{o}
$$

Here, $\mu$ denotes the absorbing coefficient. The chi signal can also be estimated via the parameters for the scattering of the photoelectrons, i.e., the EXAFS equation;

$$
\chi(k)=\sum\left[\left(N_{j} \phi_{j}(k) \varepsilon x \pi\left(-2 k^{2} \sigma_{j}^{2}\right)\right) /\left(k P_{j}^{2}\right)\right] \operatorname{oin}\left[2 k P_{j}+\delta_{j}(k)\right]
$$

and " $\mathrm{O}_{\mathrm{j}}{ }^{2}$ " is the mean-square disorder with the neighboring atom distances. Here, " $\mathrm{N}$ " is the coordination number of the neighboring atom, " $\mathrm{f}(\mathrm{k})$ " is the scattering amplitude, " $\mathrm{\delta}_{\mathrm{j}}(\mathrm{k})$ " is the scattering phase shift, " $R$ " is the distance of the neighboring atom As the scattering data is related to the photoelectrons' interactions among the atoms and it is indirectly gives information about the mean free path which means the average distance travelled by the photoelectron, i.e. $\mathrm{k} \sim 1 / \lambda$. In the absorption process, the photoelectron is created with the transferred excess kinetic energy by the photon-electron interaction mechanism. If an incident photon excites a core electron with an energy above the bond energy, the rest of the energy is used as the kinetic energy by the photoelectron to free itself from its source atom. The kinetic energy has a high reduction due to the interstitial potentials when a different type of atoms is in the neighborhood. Such kinds of materials reveals roughness in the interstitial potential where photoelectrons lost much of their kinetic energy and shorten their mean free paths. The mean free path of the photoelectron also highlights the potential distribution in the material that has an important influence on the conductivity of the material via the free electrons. In a material, the lowest product of " $\rho \times \lambda "$ is related to the highest conductivity on a metal wire [5]. 
Materials are classified as conductors, semiconductors, or insulators according to their electric charge or heat transfer capabilities. Most of the metals are conductive and the least conductive materials are called insulators such as wood, plastics, and ceramics. Best conductive metals are known as silver, copper, gold, aluminum, rhodium, etc.. There are different techniques to determine the conductivity or resistance of the metals, like 4-point probe, electrochemical analysis, Hall effect, etc..The electrical conductivity behavior of the materials is directly related to the mean free path of the free electrons. In a material, free electrons travel in the interstitial potential which obtained jointly by the atoms in the material. So from the point of view, data collected with the XAFS technique can also be used to estimate the electrical properties of the materials.

To study the single crystalline metals is useful to find out such a relation to avoid the effects of the impurities and the defects that can naturally occur in the materials. Besides, the yields of a substitution process can also provide data to enlighten the interplays between the host and the substituted atom in a crystal. In this study, boron atoms are substituted in the iron coordination to reveal its influence on bonding and chemical environment via the electronic properties. In this study, $10 \%$ boron substituted $\mathrm{CuFeO}_{2}$ oxide material was studied theoretically for its electronic and crystal structure properties.

\section{Materials and Method}

The calculations for the study are performed by the FEFF 8.2 code, which is a space multiple scattering approach [8-10]. For the calculation of the XAFS analysis, data were produced from the $\mathrm{Fe} \mathrm{K}$-edge of the $\mathrm{CuFeO}_{2}$ and $\mathrm{CuFe}_{1-\mathrm{x}} \mathrm{B}_{\mathrm{x}} \mathrm{O}_{2}$ boron substituted oxide. For the calculations, input files were generated by the TkAtoms code [11]. An iron atom in the input file was selected as the source atom, i.e. the photoelectron emitter. The input file was generated for $15 \mathrm{~A}^{\circ}$ thick $\mathrm{CuFeO}_{2}$ cluster containing 656 atoms $(\mathrm{Cu}, \mathrm{Fe}$, and $\mathrm{O})$ and 656 atoms $(\mathrm{Cu}, \mathrm{Fe}, \mathrm{B}$, and $\mathrm{O})$, where a Fe atom was chosen as the source atom. For the calculations, XRD data was created via the MAUD software which is a diffraction/reflectivity analysis code [12].

\section{Results and Discussion}

Calculated x-ray diffraction pattern data is given in figure 1 as a comparison of the boron substituted and the pure $\mathrm{CuFeO}_{2}$ oxide. The bold sketched XRD pattern has been taken from a real sample of us (that's the reason for the noisy data) and the pattern in red is calculated by the MAUD software with $10 \%$ boron substitutions in iron coordinations in the $\mathrm{CuFeO}_{2}$ cluster. 


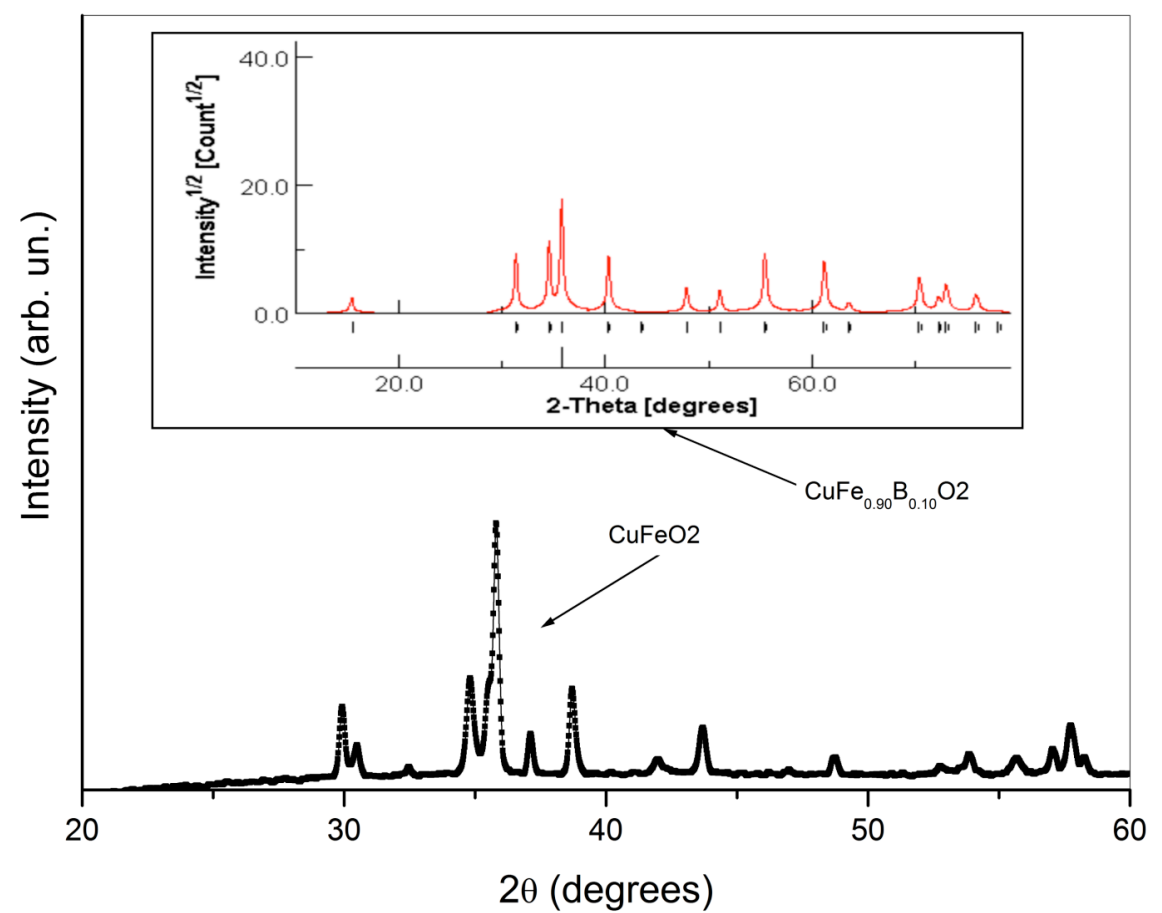

Fig. 1. Comparison for the $\mathrm{XRD}$ patterns of $\mathrm{CuFeO}_{2}$ and $10 \%$ boron substituted $\mathrm{CuFeO}_{2}$.

With the boron substitution in the iron coordination, a change in the XRD pattern peak positions was observed. No extra powerful peak formation has been observed and this means that boron atoms sit in Fe locations and preserved the crystal structure properties. To probe the influence of boron substitution on the electronic structure of its neighbors, on the iron, or on the crystal structure properties, X-ray absorption Fine Structure (XAFS) Spectroscopy study is the best choice. In Figure 2, calculated Fe K-edge XAFS spectra of the $\mathrm{CuFeO}_{2}$ and $10 \%$ boron substituted $\mathrm{CuFeO}_{2}$ material are given in comparison.

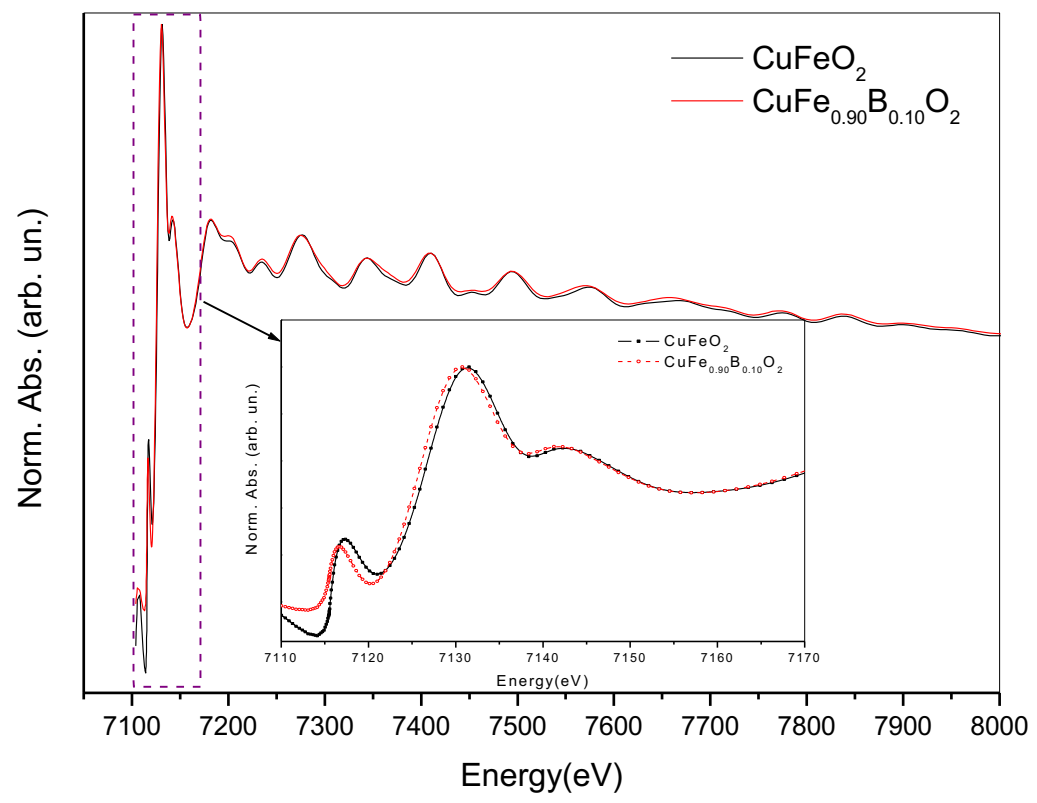

Fig. 2. Fe K-edge XAFS spectral comparison for the $\mathrm{CuFeO}_{2}$ and $10 \%$ boron substituted $\mathrm{CuFeO}_{2}$. 
A high agreement in the XAFS spectra of the materials is observed in fig.2. The symmetry of both absorption spectral features has confirmed the calculated XRD patterns. However, a slight energy shift $(0.7 \mathrm{eV})$ to the lower energy side in the boron substituted sample highlights the change in the oxidation state of the iron electronic structure. The absorption peak of the pure $\mathrm{CuFeO}_{2}$ oxide has a maximum at $7131.4 \mathrm{eV}$ and boron substituted material has the maximum at $7130.7 \mathrm{eV}$. The inset in the figure is also given to guide for the details. Fe K-edge spectra are a result of the 1 s electron transition to unoccupied $4 p$ levels in an excitation process. Actually, $3 \mathrm{~d}$ levels are also unoccupied, but due to the quantum selection rules, electronic transitions of $\mathrm{s} \rightarrow \mathrm{d}$ is dipole forbidden. Thus, $1 \mathrm{~s}$ electrons can make transitions to the $4 \mathrm{p}$ level which is located above the $3 \mathrm{~d}$ level as the main route. However, with a hybridization via $\mathrm{O} 2 \mathrm{p}-\mathrm{Fe} 3 \mathrm{~d}$ levels, quadrupolar transitions may also occur weakly. The weak peak feature just below the main edge is a result of the quadrupolar interaction and this part is called the pre-edge peak. The pre-edge peak addresses a powerful Fe-O hybridization via the strong coupling between the outer shell electrons of the neighboring iron and oxygen atoms.

Boron has the same ionic value $(3+)$ as ionic iron. However, the ionic radii of boron and iron have highly different values as; $0.023 \mathrm{~nm}$ and $0.060 \mathrm{~nm}$, respectively. Despite the confirmation of the boron locations in the iron coordination by the XRD patterns and XAFS spectra, huge difference in the ionic radii of the atoms in the substitution processes should be a problem for boron to sit in the iron coordination. The effect of the boron substitution in the crystal order of the $\mathrm{CuFeO}_{2}$ material can be best analyzed by the scattering amplitude which can be extracted from the Extended XAFS (EXAFS) part via the given equation (1). The scattering intensity graph is given in figure 3 .

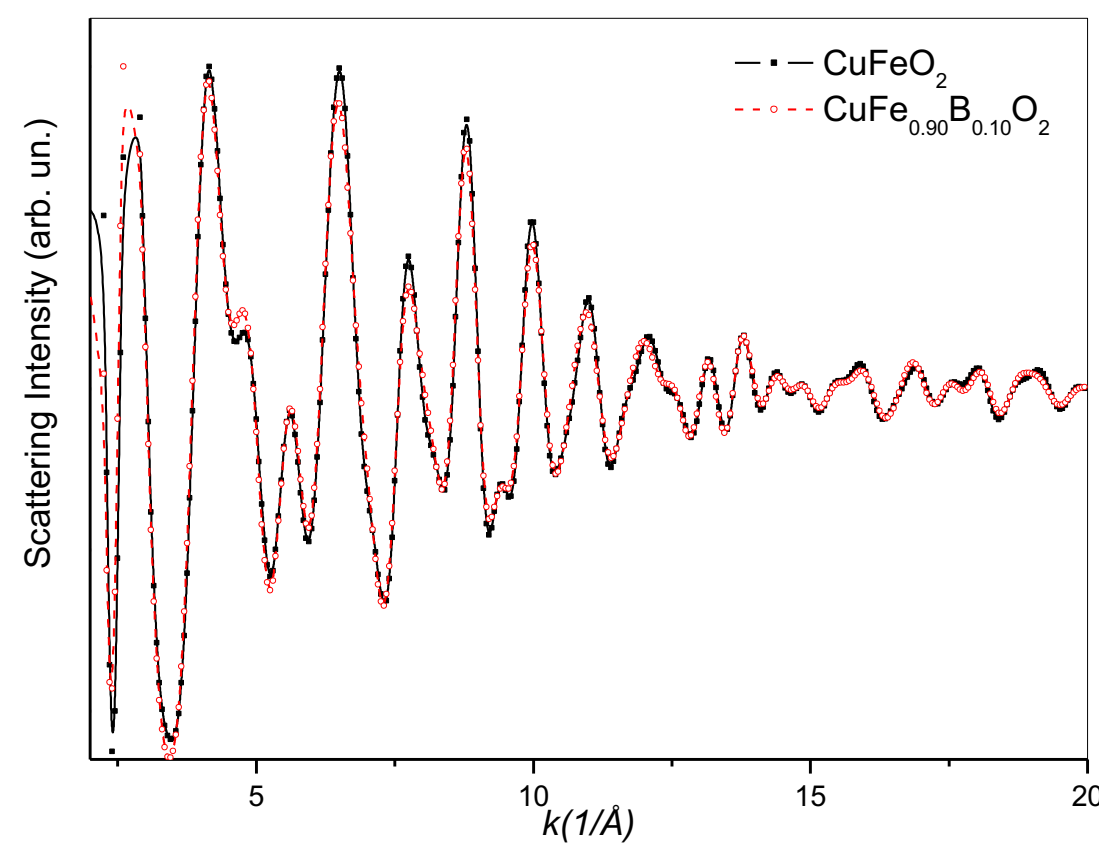

Fig. 3. EXAFS scattering intensity comparison for the $\mathrm{CuFeO}_{2}$ and $10 \%$ boron substituted $\mathrm{CuFeO}_{2}$.

In figure 3, high agreement with the peak structures and also the high symmetry in the spectra also confirms the remaining crystal structure in the material, even boron substitution. 
However, weak decays at some scattering intensities highlight the light atoms' presence in the crystal, i.e., Boron. When a substitution occurs in a material, the interstitial potential among the atoms in the material causes fluctuations for the photoelectrons where it travels. The fluctuations are due to the change in the merits of the potential of the neighboring atoms. When the substitution is taken into account for the light atoms, if it sits in the coordination of another atom, like here, the photoelectrons' kinetic energy decay is lesser than before. For heavier atoms' substitution is subject to a study, then new interstitial potential becomes fatal for the photoelectrons due to the increase in the potential borders, which are treated as barriers. The Fourier transform (FT) of the scattering intensity yields the Radial Distribution Function (RDF) of the atoms in the crystal. The RDF gives the atomic locations and distances of the atoms which are located mainly in the first and the second-row vicinity of the source atom, which is located in the origin on the $1 \mathrm{D}$ axis. In figure 4, the $\mathrm{RDF}$ of the pure $\mathrm{CuFeO}_{2}$ material is given in comparison with the boron substitute $\mathrm{CuFeO}_{2}$ material.

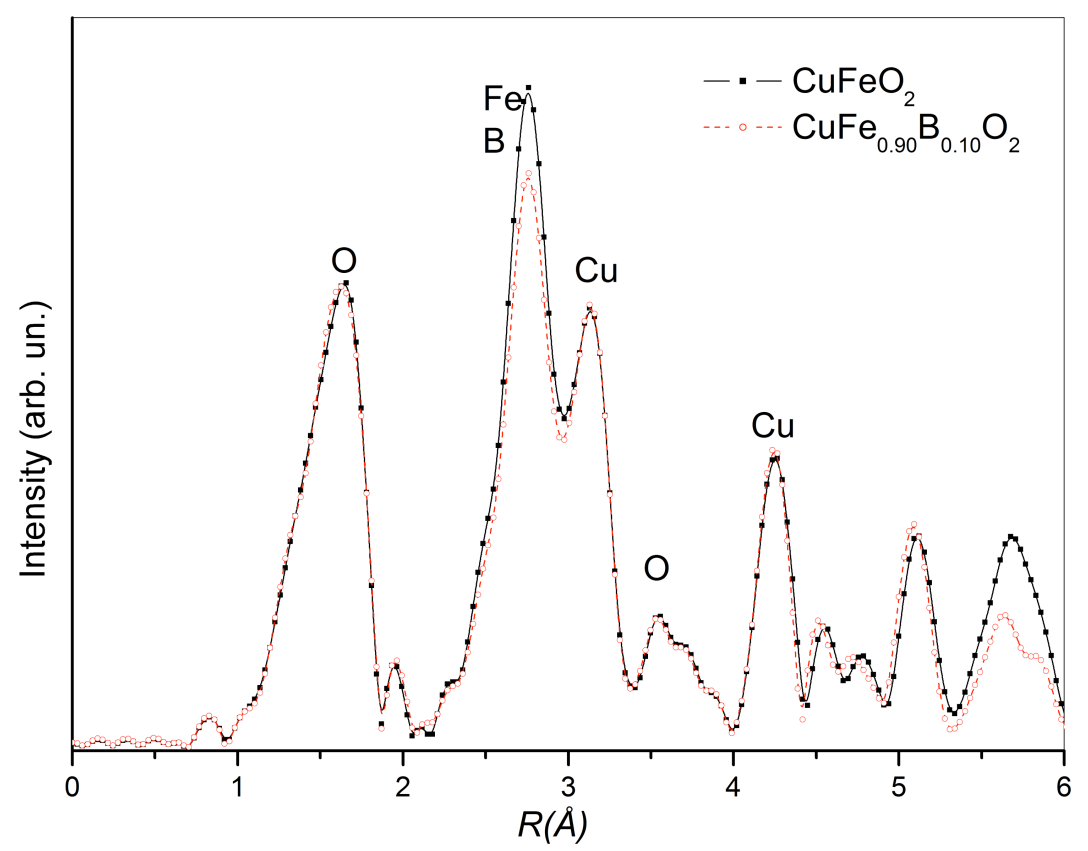

Fig. 4. Radial Distribution Function intensity of the $\mathrm{CuFeO}_{2}$ and $10 \%$ boron substituted $\mathrm{CuFeO}_{2}$.

According to figure 4, the boron sitting peaks are apparent and no need for any extra analysis to find out the boron locations. Analysis results of figure 4, the atomic distances from the source $\mathrm{Fe}$ atom which is sitting at the origin are: $\mathrm{O}$ atoms sit at $1.98 \AA$ distance from an iron atom, Fe atoms sit at $3.01 \AA$ distance and one of them is Boron. The third nearest neighbor is determined as the copper $(\mathrm{Cu})$ atom which is located at a $3.34 \AA$ distance.

\section{Conclusion}

In this study, boron atoms were substituted in the Fe coordinations. Due to the high difference in the ionic radii of the host and substituted atoms, different crystal structure formation was expected. However, calculations showed that boron atoms tend to locate in Fe coordination and prefer to the part of the host crystal and bond with the oxygen atoms strongly which are in its neighborhood. The presence of the light boron atoms was determined to weaken the 
scattering intensities which cause a longer mean free path for the photoelectrons. The longer mean free path means lower interstitial potential and hence it means a better conductivity of the material, according to the equation that gives the conductivity; i.e., $\rho \times \lambda$.

\section{References}

[1] Ozkendir, O.M., The electronic structure study of Fe L3 edge in CuFeO2, J. Optoelectron. Adv. Mater. Rapid Commun, 3, 586-591, 2009.

[2] Eerenstein, W., Mathur, N.D., Scott, J.F., Multiferroic and magnetoelectric materials, Nature, 442, 759, 2006.

[3] Ozkendir, O.M., Boron Activity in Metal Containing Materials, Advanced Journal of Chemistry, Section B: Natural Products and Medical Chemistry, 2 (2), 48-54, 2020.

[4] Ozkendir, O.M., Yuzer, A., Influence of Sm-substitution on the crystal and electronic structure of the FeBO3 oxide, Journal of Optoelectronics and Advanced Materials, 20(34), 201-207, 2018.

[5] Ozkendir, O.M., Crystal and Electronic Study of Neodymium-Substituted CuFeO2 Oxide, Metallurgical and Materials Transactions A, 47(6), 2906-2913, 2016.

[6] Thole, B.T., Carra, P., Sette, F., Van der Laan, G., X-ray circular dichroism as a probe of orbital magnetization, Phys. Rev. Lett., 68, 1943, 1992.

[7] Gall, D., Electron mean free path in elemental metals, Journal of Applied Physics, 119, $085101,2016$.

[8] Ozkendir, O.M., Crystal and Electronic Study of Neodymium-Substituted CuFeO2 Oxide, Metallurgical Materials and Metals Transaction A, 47A, 2906-2913, 2016.

[9] Ozkendir, O.M., Structural and Magnetic Study of CuxFeCr1-xO2 Oxides Under High External Magnetic Fields, J. Electron. Mater., 42(6), 1055-1062, 2013.

[11] Ankudinov, A.L., Ravel, B., Rehr, J.J., Conradson, S.D., Relativistic calculations of spindependent x-ray-absorption spectra, Phys. Rev. B, 56, R1712, 1997.

[12]Ravel, B., ATOMS: crystallography for the X-ray absorption spectroscopist, $J$. Synchrotron Radiat., 8(2), 314, 2001.

[13] Lutterotti, L., Chateigner, D., Ferrari, S., Ricote, J., Texture, residual stress and structural analysis of thin films using a combined X-ray analysis, Thin Solid Films, 450, 34-41, 2004. 